\title{
BMJ Open Cohort profile: Design and implementation of the Danish Physiotherapy Research Database for patients receiving primary care with chronic disease
}

\author{
Erhard Næss-Schmidt (D) , ${ }^{1}$ Nils-Bo de Vos Andersen, ${ }^{2}$ \\ David Høyrup Christiansen, ${ }^{3,4}$ Jørgen Feldbæk Nielsen, ${ }^{1}$ Peter William Stubbs ${ }^{5}$
}

To cite: Næss-Schmidt E, Andersen N-BdV, Christiansen $\mathrm{DH}$, et al. Cohort profile: Design and implementation of the Danish Physiotherapy Research Database for patients receiving primary care with chronic disease. BMJ Open 2020;10:e040207. doi:10.1136/ bmjopen-2020-040207

- Prepublication history and additional material for this paper are available online. To view these files, please visit the journal online (http://dx.doi. org/10.1136/bmjopen-2020040207).

Received 11 May 2020 Revised 02 September 2020 Accepted 10 October 2020

Check for updates

(C) Author(s) (or their employer(s)) 2020. Re-use permitted under CC BY-NC. No commercial re-use. See rights and permissions. Published by BMJ.

For numbered affiliations see end of article.

Correspondence to Erhard Næss-Schmidt; erhnae@rm.dk

\section{ABSTRACT}

Purpose Free of charge physiotherapy (FCP) is free physiotherapy provided by the Danish government for patients with a range of chronic diseases. To date, the population has not been described in depth making evaluation and decision making difficult. The purpose of this study was to (1) describe the development and the content of a novel clinical physiotherapy database for FCP (PhysDB-FCP) and (2) present the cohort profile based on the data collected.

Participants Ninety-nine clinics (17 460 FCP patients) were invited to participate in the development process from 2018 to 2019. Eleven clinics consented (2780 FCP patients) and 534 patients performed the physiotherapy assessment using the PhysDB-FCP tool, with 393/534 completing the patient survey.

Findings to date The content of the PhysDB-FCP was developed through an iterative process involving consensus between clinical and research workgroups. Prior to using the tool all consenting sites received training to use/administer the tool. All data were collected/stored using the PhysDB-FCP. Items finally chosen for the PhysDB-FCP included demographic information, questions about health status and daily functioning, functional tests, treatment plan and validated questionnaires. The initial patient cohort composed of $63.4 \%$ women with main diagnoses of multiple sclerosis (22.7\%) and Parkinson's disease (17.0\%). The ability to perform personal/instrumental activities of daily living and functional ability varied widely. Other non-physiotherapy related issues were identified in numerous patients (ie, $34.9 \%$ of patients were at risk of depression) and multidisciplinary interventional approaches could be considered. Future plans The current study has provided a comprehensive description of patients receiving FCP, using data collected from the novel PhysDB-FCP. Collected information can be used to facilitate microlevel to macrolevel programme evaluation and decisions. Although the PhysDB-FCP is promising, the tool requires optimisation before it is implemented regionally and/or nationally.

\section{Strengths and limitations of this study}

- We implemented and piloted a generic physiotherapy assessment tool and data collection/storage platform for patients receiving free of charge physiotherapy in a region of Denmark.

- Clinical and research staff were consulted in the development of the tool, however a variety of frontline clinicians were not consulted which may have reduced recruitment and compliance to items in the tool.

- Only 11/99 invited clinics partook in the study, however, clinics were broadly representative in terms of the size of the practice and size of the town/city served.

- 393/534 patients that received a physiotherapy assessment completed a patient survey about education, employment, general health, as well as the EQ-5D, WHODAS 2.0 and WHO-5 questionnaires.

- This cohort may be limited by the low rate of adherence at especially at follow-up which may be due to the generic assessment tool that may have been too comprehensive in a cohort with a range of patient diagnoses.

\section{INTRODUCTION}

Chronic disease covers a wide range of diagnoses and age groups with different functional ability, clinical course and prognosis. With an increased life expectancy the incidence and cost of chronic disease is becoming greater. ${ }^{1}$ As such, changes in policy and governmental interventions at different levels may be required. Worldwide, people with chronic disease have more life-affected years and will require more frequent support for activities of daily living (ADL) and societal participation. ${ }^{2}$ Clinical research in chronic disease has demonstrated that exercise improves the ability to perform $\mathrm{ADL}^{3}$ regardless of condition. Further, regular exercise 
can reduce the risk of further complications and comorbidities associated with chronic disease. ${ }^{45}$ The long-term effects of exercise and treatment may also be influenced by individual psychological and social factors, such as motivation and self-efficacy. Lack of compliance when participating in exercise programmes has been linked to lower motivation and self-efficacy among patients with chronic disease. ${ }^{6}$ Therefore, providing active continuous support in exercise programmes for people with chronic disease, and addressing psychological and social factors, may improve compliance and health outcomes.

In Denmark, patients with chronic disease are often managed by primary care physiotherapists. Denmark has a state financed healthcare system, which provides free primary care services to people with health problems. One of these services is free of charge physiotherapy (FCP), which provides ongoing care to people with chronic disease (who fulfil strict disability criteria). FCP is primarily performed by private practice physiotherapists, and referred by general practitioners. In 2016, private practice physiotherapists provided FCP to more than 67000 patients including 43 different chronic disease categories. ${ }^{7}$ Depending on the disease severity and stage, the purpose of FCP is to either (1) increase function, (2) maintain function or (3) delay/slow the loss of function. ${ }^{8}$ Patients in the programme are classified into one of two groups, entitled to different treatment depending on their level of disability; (1) patients with severe disability (defined as 'a person who cannot manage indoors for 24 hours without help or aids for daily personal life') who may receive both individual (home-based or clinic-based) therapy and group therapy (clinic-based) or (2) patients with less severe disability (defined as 'an abnormal function of the sensor-motoric or nervous-system'), which includes patients with a progressive diagnosis (such as newly diagnosed multiple sclerosis) who receive only group therapy.

Currently, physiotherapists treating patients in the FCP programme are only required to report the type and amount of treatment provided. Providing more comprehensive data (ie, data from the initial subjective assessment, results of objective assessment and through patient surveys) would be useful and provide information on the types of patients, severity of illness and treatment strategies implemented. As this information/ata are currently not reported centrally, it is difficult to evaluate the FCP programme. For private clinical practice, municipalities, organisational positions and the Danish health authorities to make informed decisions on the FCP programme, systematic reporting is required, including more details about the patients receiving the service and how these patients are managed.

In 2015, funding was allocated to the development and implementation of a standardised tool to collect and store comprehensive and systematic data of patients treated in primary care receiving physiotherapy database for FCP in Denmark (PhysDB-FCP). The PhysDB-FCP provides an up-to-date description of the population receiving
FCP including functional status, psychosocial status, goal setting, interventional aims and follow-up evaluation. In addition, the PhysDB-FCP has the potential to facilitate research, guide future decisions/directions, optimise patient care and ensure continued efficacy/efficiency of the FCP programme.

The current cohort profile aims to (1) describe the development and the content of the clinical database PhysDB-FCP and, (2) present the cohort profile based on data collected using the PhysDB-FCP tool. In addition, we will outline the potential uses and discuss possible future directions of the PhysDB-FCP.

\section{STUDY DESIGN}

The current study is a descriptive study of the development and implementation of a novel research database of patients with chronic disease receiving FCP and provides a cohort profile of the preliminary data.

\section{DATABASE DEVELOPMENT}

\section{Purpose of the PhysDB-FCP}

The purpose of the PhysDB-FCP project is to (1) provide an easy to use digital, online, structured and standardised psychosocial and functional testing tool to guide initial clinical assessments, follow-up assessments and document progress of patients receiving FCP, (2) assist in the efficient collection of comprehensive assessment data, (3) store data that is accessible, updated automatically and can be easily exported to enable evaluation and decision making of the FCP programme and (4) facilitate the use of a common language using International Classification of Functioning (ICF) terms. ${ }^{9}$

\section{Patient and public involvement}

The PhysDB is intended as a tool for physiotherapists to guide and structure their clinical assessment along with the purpose of collecting research data. As such, clinicians and researchers were used and influenced the development of the initial tool. However, the PhysDB was continuously tested on patients during this phase and adapted to increase its relevance to individual patient groups. Furthermore, as we have completed the initial development phase, and used the PhysDB in a range of patients, we are now able to consult patients to further optimise the tool for a wide range of patients. Within this, we will perform focus group discussions and semistructured interviews with patients (and therapists) to further improve the tool and provide guidance/considerations on all aspects of the tool.

\section{PhysDB-FCP development}

The PhysDB was established in 2008 to provide a digital online database to collect data on patients with only musculoskeletal problems. In 2015, a new project for patients receiving FCP of all diagnoses (PhysDB-FCP) 
was supported by funding from the Danish Physiotherapy Research and Practice fund.

The PhysDB-FCP project was initiated by six clinical physiotherapists. These physiotherapists formed a clinical workgroup that met six times between 2015 and 2016. The clinical workgroup consisted of physiotherapists, each with $>10$ years' experience in treating patients with chronic disease using the FCP service. The physiotherapist in charge of the project (NBA, with $>30$ years' experience) also met with a research workgroup eight times between 2015 and 2017. The research workgroup consisted of one researcher with expertise in rehabilitation (Defactum, Region Midtjylland), two researchers with expertise in occupational rehabilitation, patient reported outcomes and epidemiology (Occupational Medicine, Danish Ramazzini Centre, Regional Hospital West Jutland) and one researcher with expertise in neurology and rehabilitation (Hammel Neurorehabilitation Centre and University Research Clinic). The assessment was continuously trialled by the clinical workgroup using patients receiving FCP to get feedback on its feasibility and relevance. Consensus on the content of the data collection tool and PhysDB-FCP was an iterative process and reached through ongoing discussions with the clinical and research workgroups. The overall purpose of the discussions was to develop a tool that offers clinically meaningful information with outcomes that could facilitate better patient care and research.

In August 2017, the data collection tool and PhysDB-FCP were finalised and pilot data collection commenced.

\section{PhysDB-FCP procedure and content}

The PhysDB-FCP provides an online standalone platform for collection and storage of both patient and clinician reported data from a survey provided to the patient and data collected from subjective and objective physiotherapy assessment performed by the physiotherapist, guided by a proforma from the PhysDB-FCP team. According to the regional ethics commission the study did not require ethical approval but required approval from the Data Protection Agencies.

Informed consent was obtained from patients prior to enrolment in the project. The procedure was as follows. First, referral to the physiotherapist was performed by the GP. Second, the patient contacted the physiotherapy clinic and talked to a health secretary or physiotherapist who provided information about the project. Third, the patient was provided a digital identifier and consented digitally. After informed consent, the secretary registered the patient, including the referral diagnosis attained from the GP.

Patients were asked to complete an online survey prior to the initial physiotherapy assessment. The survey was also administered prior to 3-month and 6-month assessments. See figure 1 for an overview.

The data collected from the patient survey and the subjective and objective physiotherapy assessment consisted of demographic information, questions about
Invited clinics, $\mathrm{n}=99$

Number of patients receiving FCP, $\mathrm{n}=17.460$

Patients receiving home-based treatment, $n=4,119$

Patients receiving clinic-based treatment, $n=13,341$

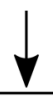

Clinics that volunteered, $\mathrm{n}=11$

Number of patients receiving FCP, $\mathrm{n}=2,780$

Patients receiving home-based treatment, $\mathrm{n}=507$

Patients receiving clinic-based treatment, $\mathrm{n}=2,273$

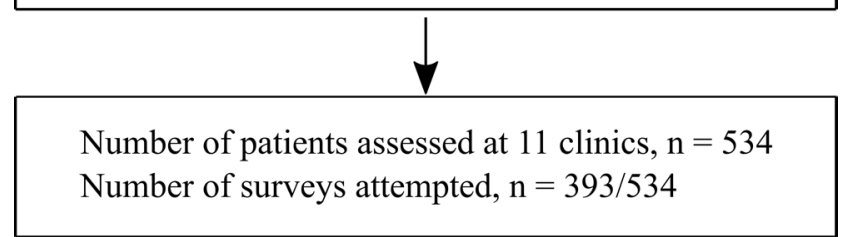

Figure 1 The process and timing of patient recruitment, performance of functional tests and administration/ completion of patient surveys. FCP, free of charge physiotherapy.

health status and daily functioning, functional tests, treatment plan and validated questionnaires. Table 1 provides an overview of the individual variables and administered questionnaires.

\section{Physiotherapy assessment}

The structured patient history covered all areas of the ICF and was guided by the clinical working group's knowledge of patients with chronic disease. The patient history took approximately $20-30 \mathrm{~min}$ to complete.

Functional performance tests were selected based the ability of the tests to track basic mobility and functioning in ADL's, in patients with a range of diagnoses. Functional tests were also selected based on the time to administer, as the total assessment should be performed in $<30 \mathrm{~min}$. The chosen tests for the lower extremities were: (1) timed up and go (TUG) (2) $30 \mathrm{~s}$ sit stand test (SST) and (3) Walking test (either $10 \mathrm{~m}, 40 \mathrm{~m}$ or $6 \mathrm{~min}$ walk test). For the upper extremity the box and block test was performed. These tests are valid and reliable for a wide range of patients. ${ }^{10-17}$

The overall treatment plan was based on the domains of ICF framework. As such, specific management of impairments, activity limitations and participation restrictions were addressed. This part was developed with input from the clinical and research working groups.

In addition, when using the PhysDB, a written journal is automatically generated containing statements and results from the patient surveys, all parts of the assessment and clinician comments. 
Table 1 Information gathered and items contained in the physiotherapy assessment and patent survey

Physiotherapy

assessment

Demographic information: name, age, sex, assessment date, year of diagnosis

Health status: pain (location and numeric rating), medication, height and mass (body mass index), involuntary weight loss and speaking/swallowing problems

Daily functioning: information about personal activities of daily living and instrumental activities of daily living, use of and type of assistive device(s), dizziness and fatigue

Obligatory functional tests

Timed up and go test, sit to stand test, walk test (choice of: $6 \mathrm{~min}, 40 \mathrm{~m}$ or $10 \mathrm{~m}$ walk test), Box and block test (if the patient has arm disability)

overall treatment plan: goal setting, overall treatment type (individual, team based, supervised or combined), expected time of treatment, expected effect of treatment (solving problem, reducing problem or delaying disease progression), patient acceptance of treatment plan planned retest assessment and disclosure to general practitioner $(\mathrm{Y} / \mathrm{N})$

$\begin{array}{ll}\text { Patient surveys } & \text { Individual questions } \\ & \text { Civil status, education, work status, number of whole/half days of sick leave (for those working), fear } \\ \text { of falling and sleep quality } \\ \text { Validated questionnaires } \\ \text { Quality of life (EQ-5D-5L), activity and participation (WHODAS 2.0-12 item), mental well-being } \\ \text { (WHO-5) }\end{array}$

\section{Optional items in the physiotherapy assessment}

Due to the diversity of diagnoses included in the PhysDB-FCP, more individualised optional assessments were required for some patients. Optional functional tests included: (1) functional analysis and postural control (Note: This was used for severely disabled patients who met certain inclusion criteria.), (2) Berg balance scale, (3) arm flexion test, (4) accelerometry to monitor daily activity, (5) full neurological examination, (6) range of motion and strength assessment, (7) Mini-Mental Stata Examination (MMSE) (cognitive assessment), (8) Tandem stance test, (9) Aastrand's 1 point test.

\section{Patient questionnaires}

The patient surveys asked general information from the patient and included validated questionnaires. Information gathered using the patent survey included civil status, education, works status, sick days (for those working), fear of falling and sleep quality (table 1, online supplemental table S1).

A separate process was used to find generic validated patient questionnaires to collect data based on the ICF domains and psychosocial factors. This process was performed by the Occupational Medicine, Danish Ramazzini Centre. The internal report recommended three tools for the PhysDB-FCP; EQ5D, WODAS 2.0 and WHO5. ${ }^{12}$

The EQ-5D (EuroQol) was chosen to measure healthrelated quality of life. The EQ-5D allows the assessment of the effect of a treatment at the impairment and activity level. It is also a valid and reliable assessment tool for a range of diagnoses. ${ }^{13}$

The WHODAS 2.0 (12 items) was chosen to measure the ICF domains in patients. Although other generic health status instruments can also be transferred to the ICF, these do not clearly distinguish between patient reported (subjective) measures of impairment, activity limitations and participation restrictions. Furthermore, it assesses disability and is used worldwide. As such, using this tool can facilitate comparisons between countries. ${ }^{14}$

The WHO-5 was chosen to assess mental well-being. It is a five-item questionnaire which assesses patient reported mental health in the preceding 2 weeks. The WHO-5 has been tested in various populations and in several countries. ${ }^{15}$ The tool is easy to apply, valid and reliable. ${ }^{15}$

\section{Modifications}

During data collection, the patient survey and clinical assessment were modified as some important questions were excluded initially. Through the pilot phase, although no questions/items were removed, some questions/items were added. Items added to the patient survey were the items; smoker, alcohol intake amount, physical load at work (if working), physical load during leisure time activities, self-perceived rating of physical fitness and desire to be more active. Items added to the physiotherapy assessment included the; modified Rankin scale score, types of leisure activities, presence of comorbidities and type of comorbidities (using an adapted list from Charleston's comorbidity index) and frequency of falls within the last month. Most items have been added to ensure the data will be comparable to other databases, and to assist in the description of general patient health and functioning. Due to the late implementation of these tools, data are incomplete and have not been reported.

\section{COHORT DESCRIPTION \\ Participating clinics}

Private outpatient clinics that provide FCP in the Region of Mid Jutland in Denmark ( $\mathrm{n}=99$ clinics) were invited to participate from August 2017 to January 2019. Figure 2 shows the total number of patients receiving FCP associated with the invited clinics. Clinics were contacted via email by the PhysDB-FCP implementation staff and invited to participate in the pilot study. The email provided 


\section{Pre-consultation}

Patient referred by the General Practitioner to FCP

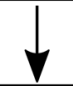

Physiotherapy clinic registers the patient and sends out the survey prior to the first consultation

\begin{tabular}{l}
\hline Patient answers the survey online before consultation \\
First consultation \\
$\begin{array}{l}\text { If survey is not answered, a short version can be } \\
\text { performed by the Physiotherapist } \\
\text { Physiotherapist performs the patient history, } \\
\text { Fonctional performance tests and goal setting }\end{array}$ \\
\hline \\
Patient performs surveys at 3 and 6 months \\
Functional performance retested at 3 and 6 months \\
\hline \multicolumn{1}{|c|}{ Intervention / treatment } \\
\hline
\end{tabular}

Figure 2 Flow chart showing the number of invited clinics, the number of clinics that volunteered, with associated number of free of charge physiotherapy (FCP) patients. Also included are the number of patients that were assessed by a physiotherapist using the physiotherapy database for FCP tool and the number of those patients that attempted the survey.

information about the research project including the purpose of the assessment tool and the potential usefulness for both clinicians and researchers. If a clinic did not reply within 4 weeks, a follow-up email was sent. If a clinic still did not reply, no further contact was made. In total, 11 private outpatient clinics agreed to participate in the pilot phase (figure 2). Following clinic agreement, a member of the PhysDB-FCP implementation staff visited the site to discuss implementation and expectations. During the visit, physiotherapists were provided a 3-hour training session on how to administer the tool and use the PhysDB-FCP software. After an introduction to the PhysDB-FCP, physiotherapists began enrolling patients. Clinics were advised to contact the PhysDB-FCP implementation group if there were any questions/troubleshooting issues.

\section{Participants}

Patients that attended the participating clinics were invited to be assessed with the PhysDB-FCP data collection tool. Inclusion criteria were: (1) $\geq 18$ years old, (2) chronic disease and an approved referral for FCP from a general practitioner and (3) being able to transport herself/ himself to and from the outpatient clinic.

\section{Statistics}

Descriptive statistics were used to describe baseline characteristics of the cohort. PhysDB-FCP. The number of patients and corresponding percentage (of the total sample) has been reported for all demographic and diagnostic information, health status variables, daily functioning and functional tests. Data management is performed using STATA V.15. No statistical comparisons have been made as data are used to describe the framework to facilitate discussion on future perspectives of the database.

\section{COHORT CHARACTERISTICS}

In total, the 11 clinics provide consultations to 2780 patients receiving FCP (figure 2). Over the period of the study, 534 FCP patients were assessed by a physiotherapist using the PhysDB-FCP tool and 393 of these patients completed the patient survey (figure 2). At 3 months, 142 patients were assessed by a physiotherapist and 179 patients answered the patient survey and at 6 months, 52 patients were assessed by a physiotherapist and 166 answered the patient survey.

The 11 clinics that volunteered represented small and large cities and similar to the 88 clinics not participating. Likewise, the number of physiotherapists working at the clinics ranged from 3 to 9 and was generally representative of the other 88 clinics however, very large clinics (>10 physiotherapists) were not represented in our study (online supplemental table S2).

\section{Characteristics PhysDB-FCP}

Demographics and characteristics of the patients assessed are reported in table 2. The majority of patients in the FCP programme were women $(63.9 \%)$, the number of patients increased with age and more patients were categorised as having severe disability $(68.4 \%)$ rather than less severe disability (30.5\%). Primary diagnoses were multiple sclerosis, Parkinson's disease, stroke and chronic arthritis (table 2). The breakdown of the 'other' diagnoses are presented in online supplemental table S3.

\section{Functional ability and activities of daily living: clinical assessment}

Data collected by physiotherapists on functional ability and ADL are reported in tables 3 and 4 . Additional data are reported in online supplemental table S4.

Table 3 provides information on the functional ability of patients. A large percentage of patients did not perform/were unable to perform the functional tests (TUG (31.1\%), 30 s STS test $(25.7 \%)$ and patients performing at least one walking test $(34.6 \%))$. Some patients $(7.9 \%)$ required modifications to complete the 
Table 2 Demographic and basic medical information gathered by the physiotherapy assessment and patient surveys

\begin{tabular}{|c|c|}
\hline Characteristics and demographic information & $\begin{array}{l}(n=534) \\
n(\%)\end{array}$ \\
\hline \multicolumn{2}{|l|}{ Gender } \\
\hline Female & $341(63.9)$ \\
\hline Male & $193(36.1)$ \\
\hline \multicolumn{2}{|l|}{ Age (years) } \\
\hline $18-29$ & $24(4.5)$ \\
\hline 30-39 & $35(6.6)$ \\
\hline $40-49$ & $75(14.0)$ \\
\hline $50-59$ & $90(16.8)$ \\
\hline $60-69$ & $139(26.0)$ \\
\hline $70-79$ & $144(27.0)$ \\
\hline $80+$ & $27(5.1)$ \\
\hline \multicolumn{2}{|l|}{ Diagnosis } \\
\hline $\begin{array}{l}\text { Multiple sclerosis (and other demyelinating } \\
\text { diseases) }\end{array}$ & $121(22.7)$ \\
\hline $\begin{array}{l}\text { Parkinson's disease (and other diseases of the } \\
\text { basal ganglia) }\end{array}$ & $91(17.0)$ \\
\hline Stroke (ischaemic and haemorragic) & $51(9.6)$ \\
\hline Chronic arthritis (ie, rheumatoid arthritis) & $46(8.6)$ \\
\hline Other & $208(39.0)$ \\
\hline Missing & $17(3.2)$ \\
\hline \multicolumn{2}{|l|}{ Type of treatment } \\
\hline One-on-one treatment (more severe) & $365(68.4)$ \\
\hline Group therapy (less severe) & $163(30.5)$ \\
\hline Missing & $6(1.1)$ \\
\hline \multicolumn{2}{|l|}{ Years with the condition } \\
\hline $0-4$ & $125(23.4)$ \\
\hline $5-9$ & $109(20.4)$ \\
\hline $10-14$ & 65 (12.2) \\
\hline $15-19$ & $57(10.7)$ \\
\hline $20+$ & $137(25.7)$ \\
\hline Missing & $15(2.8)$ \\
\hline
\end{tabular}

See online supplemental material for further breakdown.

STS (ie, lowered seat height, assistive devices) however the type of modification wasn't routinely collected. Most patients $(94.9 \%)$ did not perform the box and block test.

Table 4 reports the type and amount of personal assistance required for patients to perform ADL. Assistance with PADL's was required by $26.4 \%$ of patients. When help with PADLs was required, this was most often provided by a partner. Assistance with IADL's was required by $47.8 \%$ of patients, with $31.1 \%$ requiring help with $\geq 2$ IADL's. Assistive devices were required by $58.1 \%$ of patients (table 4 ) with $32.8 \%$ of patients requiring $\geq 2$ assistive devices (online supplemental table S4). The types and number
Table 3 Information on functional performance gathered by physiotherapists

\begin{tabular}{|lc}
\hline Functional ability & $\begin{array}{l}\text { All patients } \\
\mathbf{( n = 5 3 4 )} \\
\mathbf{n}(\%)\end{array}$ \\
\hline TUG & \\
\hline Not performed/unable to perform & $166(31.1)$ \\
\hline Performed & $368(68.9)$ \\
\hline Time $(\mathbf{s})$ & $(n=368)$ \\
\hline$<4.99$ & $39(10.6)$ \\
\hline $5-9.99$ & $217(59.0)$ \\
\hline $10-19.99$ & $82(22.3)$ \\
\hline $20-29.99$ & $19(5.2)$ \\
\hline$>30$ & $10(1.9)$ \\
\hline STS in 30s & \\
\hline Not performed/unable to perform & $137(25.7)$ \\
\hline Performed with modifications & $42(7.9)$ \\
\hline Performed without modifications & $355(66.5)$ \\
\hline Number of STS & $(n=397)$ \\
\hline 1-3 & $6(1.5)$ \\
\hline $4-6$ & $40(10.1)$ \\
\hline $7-9$ & $89(22.4)$ \\
\hline $10-12$ & $119(30.0)$ \\
\hline $13+$ & $143(36.0)$ \\
\hline Walking tests & $185(34.6)$ \\
\hline Not performed/unable to perform & $348(65.2)$ \\
\hline Performed one test & $1(0.2)$ \\
\hline Performed two tests & $99(18.5)$ \\
\hline 10MWT & $138(26.8)$ \\
\hline $40 M W T$ & $113(21.2)$ \\
\hline $6 M W T$ & $507(94.9)$ \\
\hline Not performed & $27(5.1)$ \\
\hline Performed & \\
\hline
\end{tabular}

6MWT, $6 \mathrm{~m}$ walk test; 10MWT, $10 \mathrm{~m}$ walk test; 40MWT, $40 \mathrm{~m}$ walk test; STS, sit to stand; TUG, timed up and go.

of assistive device(s) used are described in online supplemental table S4.

\section{Functional ability and activities of daily living: surveys}

A total of $393 / 534$ patients attempted the surveys. The results from the patient surveys are shown in online supplemental table S4. The majority of patients were on a pension/retired $(51.9 \%)$ or employed with or without special arrangements (23.6\%), lived in a house (60\%) or apartment $(23.4 \%)$, with $70.4 \%$ living with another person and $25.2 \%$ living alone. $375 / 393$ patients $(95.4 \%)$ completed the EQ-5D-5L and 320/393 patients $(81.4 \%)$ completed the WHODAS 2.0 and WHO-5. 
Table 4 Assistance required when completing activities daily living gathered by physiotherapists

\begin{tabular}{lc}
\hline Assistance with ADL's & (n=534) \\
$\mathbf{n}(\%)$
\end{tabular}

*Some patients receive help from more than one source and total percentage will be greater than $100 \%$.

†Some patients receive help for more than one activity and total percentage will be greater than $100 \%$.

$\ddagger$ See online supplemental material for further breakdown. IADL, instrumental activities of daily living; PADL, personal activities of daily living.

For the EQ-5D-5L (figure 3), only 8/375 participants reported no problems in all five domains. The level of problems for patients varied with the domain. The majority of patients had no problems with self care $(60.5 \%)$ and anxiety/depresison (63.5\%) however for

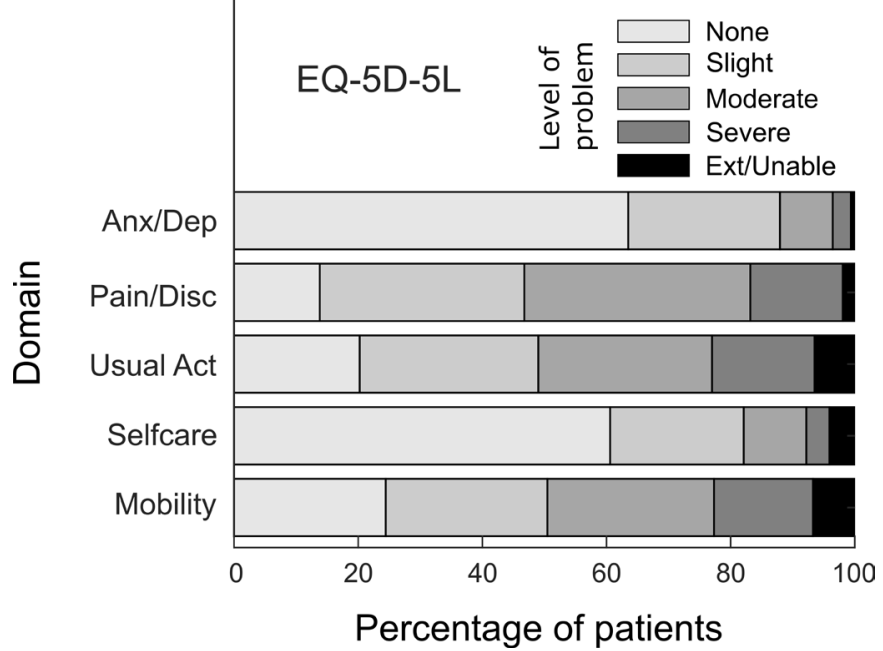

Figure 3 Summary results for the EQ-5D-5L questionnaire results. The percentage of patients at each problem level for each domain is shown in the horizontal bars (range 0\%$100 \%)$. The level of the problem was graded from no problem (none, lightest shade of grey) to extreme problems/unable to perform (Ext/unable, black). Domains measured by the EQED-5L were amount of anxiety/depression (Anx/Dep), amount of pain/discomfort (Pain/Disc), difficulty performing usual activities (Usual Act), difficulty washing or dressing (Selfcare) and difficulty walking (Mobility).

mobility, usual activities and self-care only 24.3\%, $20.3 \%$ and $13.9 \%$ reported no problems, respectively.

For the WHODAS 2.0 (figure 4), no patients reported 'no difficulty' in all 12 domains. Most patients had at least some issues with emotional affect due to their condition $(83.4 \%)$, standing for long periods $(79 \%)$, walking a long distance $(75.2 \%)$ and household responsibilities $(73.9 \%)$. Patients had least issues with washing their whole body $(35.0 \%)$, dealing with people they do not know (social, $37.6 \%)$, maintaining friendships $(40.0 \%)$ and concentrating on something for $10 \mathrm{~min}(42.9 \%)$.

The WHO-5 results are shown in figure 5. Scores were converted into a total score $/ 25,{ }^{15}$ and expressed as a percentage, with $0 \%$ representing worst imaginable wellbeing and $100 \%$ representing best imaginable well-being. For the purpose of the current study, patients at high-risk, moderate-risk and low-risk of depression was defined by scores ranging from $0 \%$ to $28 \%, 29 \%$ to $50 \%$ and $>50 \%$, respectively. ${ }^{15}$ The percentage of patients at high-risk, moderate-risk and low-risk of depression were $12 \%$, $22.9 \%$ and $65.1 \%$, respectively.

\section{FINDINGS TO DATE}

This study describes the pilot process for the Danish physiotherapy database for patients receiving FCP (PhysDB-FCP). The intention of the PhysDB-FCP is to create an easy to use assessment tool to record, monitor, assess and reassess patients with chronic disease receiving FCP. This study has described the development process, the content of the assessment tools and the cohort characteristics. We have illustrated that it is possible to provide a detailed 


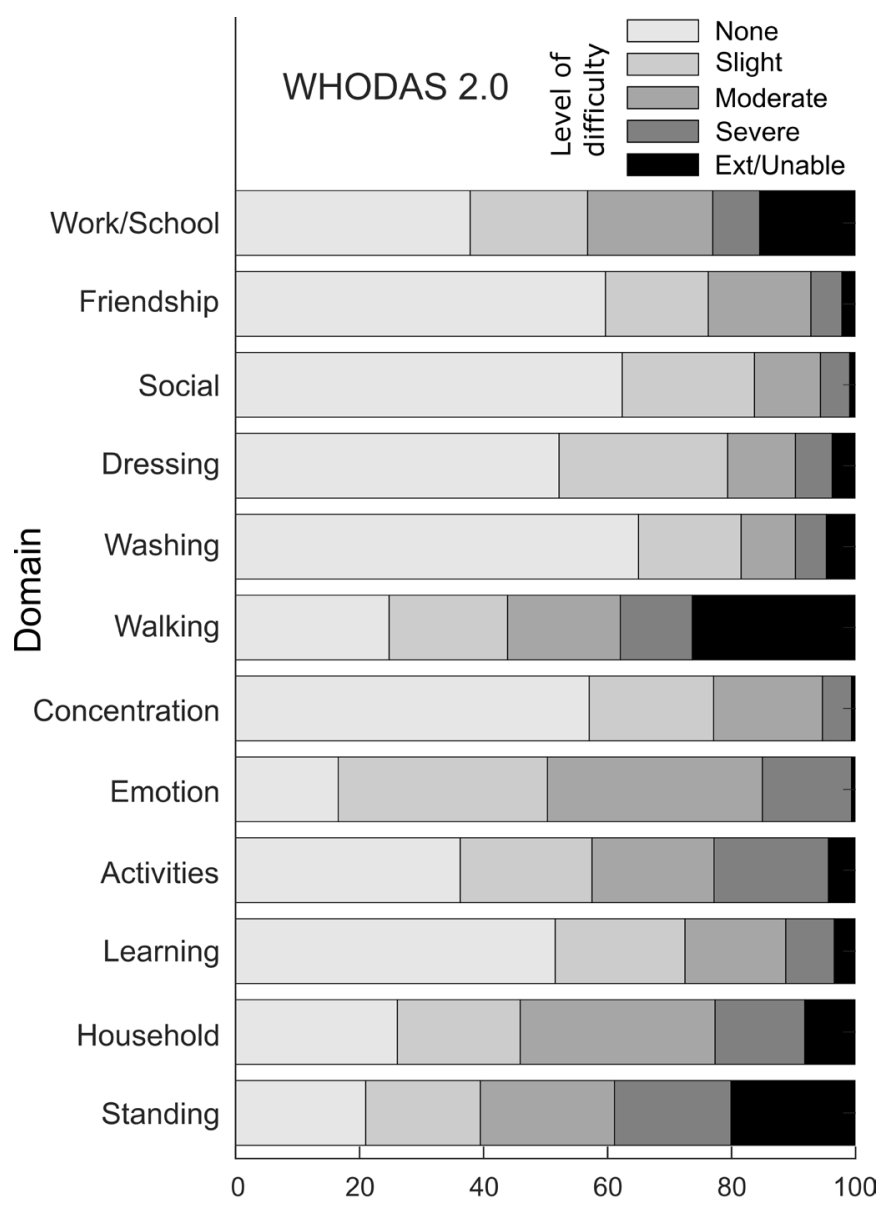

Percentage of patients

Figure 4 Summary results for the WHODAS 2.0 questionnaire results. The percentage of patients at each level for each domain is shown in the horizontal bars (range $0 \%-100 \%)$. The level of difficulty was graded as the difficulty in performing each item in the past 30 days, from no difficulty (none, lightest shade of grey) to extreme difficulty/unable to do (Ext/unable, black). Domains measured by the WHODAS 2.0 were the level of difficulty in standing for long periods (Standing), difficulty taking care of household responsibilities (Household), difficulty learning a new task (Learning), problems joining community activities (Activities), emotional affectedness by health problems (Emotion), difficulty concentrating (Concentration), difficulty walking a long distance (Walking), difficulty washing the body (Washing), difficulty getting dressed (Dressing), difficulty dealing with people (Social), difficulty maintaining friendship (Friendship) and difficult yin day-to-day work (Work/School).

description of these patients. Such a description has not been performed previously for patients with chronic disease receiving FCP, and as such, the PhysDB-FCP provides the possibility for future research investigating long-term interventions in patients with chronic disease. Despite this, the initial data also indicate that the PhysDB-FCP and associated assessment and survey, require further development.

\section{Content of the PhysDB-FCP and comparisons with other} databases

The PhysDB-FCP has the potential to provide extensive data on patients receiving FCP. Nationally, similar

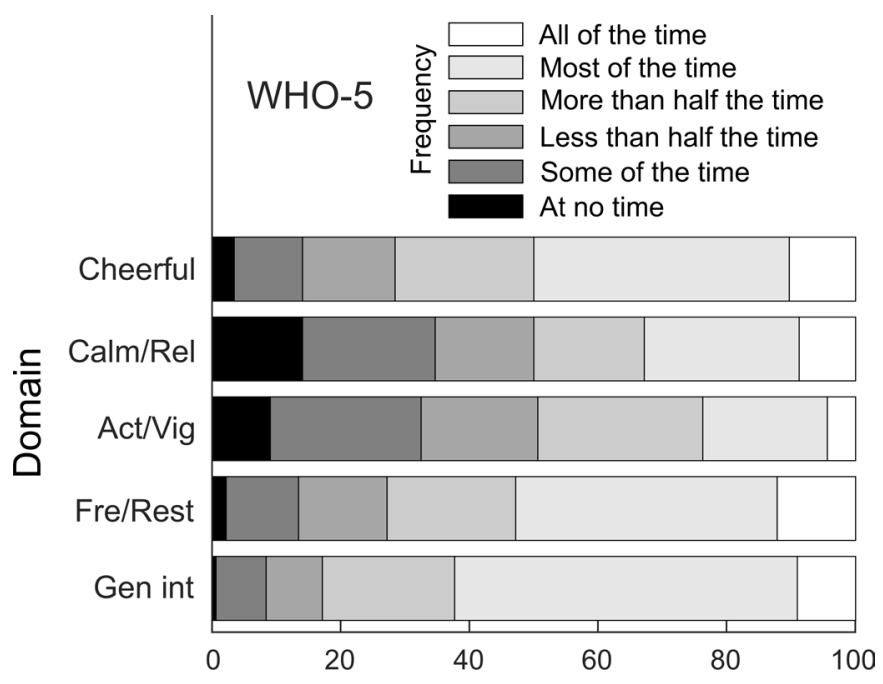

\section{Percentage of patients}

Figure 5 Summary results for the WHO- 5 questionnaire results. The percentage of patients at each problem level for each domain is shown in the horizontal bars (range $0 \%-100 \%)$. The frequency of feelings in the past 2 weeks was graded from 'All of the time' (white) to 'At no time' (black). Domains measured by theWHO-5 were 'Over the past few weeks ...'; 'I have felt cheerful and in good spirits' (Cheerful), 'I have felt calm and relaxed' (Calm/Rel), 'I have felt active and vigorous' (Act/Vig), 'I woke up feeling fresh and rested'(Fre/Rest) and 'my daily life has been filled with things that interest me' (Gen int).

registries have been created for specific diagnosis groups such as the Danish stroke registry (for patients with acute stroke ${ }^{16}$ ) and the Danish Head Trauma Database (for patients with severe traumatic brain injury ${ }^{17}$ ) and databases specific to inpatient hospital care (such as those used in ${ }^{18-20}$ ). Internationally, databases have been implemented in areas of primary care (such as the FYSIOPRIM Trondheim $^{21}$ ), patient reported outcomes measurements in musculoskeletal care (Dutch physical therapy quality programme $e^{22}$ ), physical therapy outcomes registry for patient of diverse diagnoses, impairments and activity limitations ${ }^{23}$ and musculoskeletal health including the National Information Service for Allied Healthcare (in the Netherlands), Maccabi Healthcare Services (in Israel) and Focus On Therapeutic Outcomes (in the USA) ${ }^{24}$ National databases are important and have been instrumental in providing guidance to policy makers to continually improve practice. These can also determine and compare practices in different rehabilitation settings and provide an indication of the success/failures of a healthcare system. These databases are often specific to a diagnosis or population type and very rarely cater to multiple physiotherapy types (ie, musculoskeletal and neurological populations). Given this, although the framework for the implementation could be used as guidance, it was not possible to use these studies for guidance on the actual content and assessments for a generic patient populations with multiple diagnoses and severities. Although databases often provide comprehensive 
general information about, they sometimes lack specific information related to ADL, specific functional scores and psychosocial measures. Prior to the allocation of funding to the PhysDB-FCP, there was a lack of clinical data on patients receiving outpatient physiotherapy for chronic conditions through the FCP scheme. With this database, we now have the ability to investigate detailed functional performance, ADL and other psychosocial factors directly related to long-term physiotherapy management. Moreover, we can assess the change in these factors in specific patient groups over time. These data may therefore have the potential to inform clinical and policy decisions.

\section{General cohort characteristics}

The PhysDB-FCP database provides a description of the initial cohort of people with chronic disease receiving FCP. This is the first time that this population has been described in Denmark, in terms of functional ability, ADL and psychosocial factors. The results indicate the diversity of patients within the FCP programme. More women than men were in the programme which broadly reflects the proportion of women and men within the main chronic disease categories. ${ }^{25-28}$ The four largest patient groups contained in the database were stroke, multiple sclerosis, Parkinson's disease and chronic arthritis. Although patients of all ages were represented, more older patients were included, which is largely consistent with previous reports of age in patients with chronic disease $\left(\mathrm{eg},{ }^{29-32}\right)$.

Unlike most databases, the PhysDB-FCP combines data from physiotherapy assessments and patient surveys. This combination in a large database provides unique opportunities to use data with a holistic view of the patient, that would normally not be collected or contained in databases. In general, the initial cohort showed higher physical functioning than expected. It was surprising how many patients required no help with PADL's $(67.0 \%)$ and IADL's (43.3\%). In addition, the functional ability of numerous patients was high with $47.9 \%$ of patients performing TUG in $<9.99 \mathrm{~s}$ and $49.0 \%$ of patients performing the 10+ STS in $30 \mathrm{~s}$. Given this, a large percentage of patients receiving FCP were high functioning in terms of mobility, despite the majority (68.4\%) being severely affected and eligible for oneon-one ongoing physiotherapy management. However, the surveys indicate that patients were struggling with other aspects of life, which were not captured by the physiotherapy assessment. This highlights that patients have other issues and provides the basis for targeting aspects of patient care other than physical therapy. This holistic overview provides the opportunity to ensure appropriate management of the patient, as well as the potential to refer the patient to clinicians in more specialised areas, or different medical areas (ie, clinical psychology). For example, $34.9 \%$ of patients were at moderate-risk or severe-risk of depression. Such information is important to understand during a treatment session as for optimal outcomes related physiotherapy, these patients may need to be medicated or undergo specific therapy targeting depression. This type of information may have been missed with a standard physiotherapy assessment.

For higher functioning patients, it may be useful to track periods were the patient is able to attend group therapy, or perform self-management strategies, home exercise programmes ${ }^{33}$ and tele-rehabilitation. ${ }^{34}$ Appropriate use of the PhysDB-FCP could help inform decision making on these strategies along with guidance for appropriate treatment or referral, while also meeting future challenges of an increased population with chronic disease, without compromising patient care.

\section{Difficulties encountered in design and implementation}

There were several difficulties encountered during the design and implementation of the database. One main issue, is that we may not have developed a solid enough implementation framework for the design, implementation and piloting of the tool, a priori. A framework such as the implementation model proposed in Meerhoff $e t$ $a l,{ }^{22}$ with the inclusion of implementation scientist in the project team, would have likely mitigated some of the difficulties we encountered (see later).

Due to the diversity of patients included in the PhysDB-FCP, and that there is currently no core outcome set for such a diverse group of patients, designing a tool that was generic enough to be relevant for all included diagnosis and multiple severities, was difficult. In an attempt to create a generic tool for all patients, the working group may have lost sight of the usability of the tool clinically. Although patients, clinicians and researchers were consulted in the design of the tool, and the questions within, it may have been beneficial to attain the views of multiple other stakeholders (ie, Implementation scientists and a wider variety of patients and frontline clinicians), outside of the core group. Such insights, prior to the implementation of the pilot process, may have improved the number of (1) sites included, (2) patients sampled at the sites, (3) patients followed up at 3 and 6 months and (4) patients that completed the patient survey.

Clinicians did not complete all assessments, and within the data extraction form there was no place to provide feedback as to why the task was not selected. For example, of the 166 patients that did not perform the TUG test, $83.7 \%$ of these patients did not perform a walking test. Although $34.9 \%$ were using a wheelchair in daily life, $48.8 \%$ (83.7\% minus $34.9 \%$ ) could walk but didn't perform the TUG. Given this, it was difficult to ascertain (1) if the task was too difficult for the patient (2) if the task was too easy for the patient (and the test was irrelevant) or (3) or for some other reason (ie, time constraint's). Embedding the potential for feedback within the data collection tool, when tests weren't performed would have provided a more comprehensive view of the functionality of the patient. Another assessment that was rarely performed was the box and block test. Although 26.4\% of patients required assistance with PADLs (and the box and block test may be indicated) only $5.1 \%$ of clinicians 
performed the test. From the current data collection platform, we do not know why this occurred. It may have been that the physiotherapist lacked the skills to perform this test, they may have felt that it was irrelevant or some other reason.

Possible other reasons for low adherence could be (1) the PhysDB data collection tool and patient survey were too long and need to be shortened for some patients, (2) more individualised assessments based on diagnosis and severity should be implemented in the tool which would provide a better overview of specific patients, (3) difficulty in tool administration as it was not embedded in their normal software system meaning clinicians had to perform 'double work' (ie, using their own system and the PhysDB-FCP system), (4) follow-up assessments at 3 and 6 months were not always relevant for patients at the sampled time points because of the chronicity of their health conditions that do not always change as quickly over time and (5) the tool was not mandatory and participating physiotherapist could chose not to use the PhysDB-FCP if circumstances weren't ideal for data collection.

To further expand on these hypotheses and elucidate processes and issues that may have caused low adherence, we are now designing a study to investigate the clinician and patient views of the PhysDB-FCP tool to formally document experiences of clinicians and patients and iterate the tool to ensure it is optimised for future use.

\section{STRENGTHS AND LIMITATIONS}

The PhysDB-FCP provides a comprehensive biopsychosocial assessment of patients which may guide clinicians in daily patient care and provide the possibility to track patients over time. This allows for the platform to be used in quality improvement, larger interventional studies, prognostic modelling and provide information for national (between region) and international comparisons in healthcare, as has been done previously for musculoskeletal conditions. ${ }^{24}$ Further, data from PhysDB-FCP can be linked to other public registries in Denmark using the national CPR system to assist in changing and enhancing patient care and outcomes. Moreover, the tool provides an assessment guide and can automatically insert data into patient records. This may facilitate equal healthcare throughout the country, aid in the collection of essential generic information and reduce consultation time. The current study provides a glimpse of the potential of the PhysDB-FCP with preliminary description of the cohort providing the basis for conversations around the patient management in the FCP programme. Despite the advantages of the PhysDB-FCP, the initial results indicate that there are some unsolved challenges.

Only $10 \%$ of the invited clinics chose to participate and therefore our sample may not be representative of all clinics in Denmark. Despite this, geographical placement and the number of small and large clinics did not differ substantially between participating and non-participating clinics (see online supplemental table S2). Adherence to the assessment and survey was lower than expected, at both baseline and follow-up, especially in a group of therapists that volunteered to be part of the programme, where higher adherence might be expected. Lower than expected adherence has also been observed in another study attempting to implement a similar database in musculoskeletal primary care physiotherapy. ${ }^{35}$ Adherence/compliance data in the current study may actually reflect a 'best case' scenario and bias the results to a more favourable adherence/compliance rate. Given this, consideration must be given to the reasons therapists may have been non-compliant. Possible reasons have been stated in the 'difficulties encountered in design and implementation' and include irrelevant assessments for many patients with a tool that is too long, sampling too frequently for chronic conditions, non-mandatory data collection form, 'double entry' of assessments in their standard assessment for and the PhysDB-FCP. Solutions to increase compliance/adherence could include (1) collecting assessment data yearly to reflect the clinical course and relevance in patients with chronic conditions, (2) embedding the PhysDB-FCP platform with the current computer programmes used in the clinic to avoid double entry (3) using a shorter generic version and adding more disease specific items or questionnaires for certain patients that may increase meaningfulness of the tool for each patient. The next step will be to collect feedback and experiences from clinicians and patients to further improve the PhysDB-FCP tool and patient survey. In a future study, we will conduct surveys, semi-structured interviews and focus group discussions with therapists and patients involved in the project to further refine and develop the PhysDB-FCP assessment tool and patient survey. In this, we intend to describe the barriers and facilitators (including how these assessments should be altered) to optimise and refine the assessment forms and questions within. Following this, therapists and patients will be re-consulted to ensure the correct changes have been implemented. In the concurrent study, patients and therapists will be asked about the potential adaptions in attempt to increase adherence. Clinicians and patients will also be asked how to create a more patient-centred assessment form.

\section{CONCLUSION}

The current study has provided a description of the development and content of the PhysDB-FCP tool for chronic patients using the FCP programme. Within this process we have collected data on an initial cohort using the tool and demonstrated that it is possible to provide a comprehensive cohort profile using data collected from the physiotherapist and patient. Although, the PhsyDB-FCP is promising, further research into the PhysDB-FCP is required to optimise the physiotherapy assessment and survey questions and to understand the barriers and facilitators to using the PhysDB-FCP from a user (therapist 
and patient) perspective. If these steps are taken and the PhysDB-FCP is implemented regionally or nationally, such data would be useful for describing the types of patients within the FCP programme and can be used to optimise the FCP programme. In turn, decisions can be made to optimise patient care.

\section{COLLABORATION}

Data available includes the data described within the text and online supplemental material. If research groups have a question they would like to answer using these data, they should apply to Nils-Bo de Vos Andersen with a brief (one page) project proposal. If the project is deemed potentially suitable (ie, a similar proposal is not already being considered and the project is within scope), meeting(s) will be scheduled with members of the project team to discuss the protocol in more detail. Following this, documents will need to be prepared by the research group proposing the project. This will include, (1) ethical approval from the home institution, (2) data sharing agreement with the Danish Data protection agency, (3) data sharing agreement with the EU. Approval processes can take up to 1 year. If data linkage with other registries is required, further approvals will be required extending the likely timeframe. In all circumstances, data released will be anonymous.

\author{
Author affiliations \\ ${ }^{1}$ Hammel Neurorehabilitation Centre and University Research Clinic, Aarhus \\ University, Hammel, Denmark \\ ${ }^{2}$ Primary Health Care and Quality Improvement, Viborg, Denmark \\ ${ }^{3}$ Occupational Medicine, Danish Ramazzini Centre, Regional Hospital West Jutland - \\ University Research Clinic, Aarhus University, Herning, Denmark \\ ${ }^{4}$ Department of Clinical Medicine, Health, Aarhus University, Aarhus, Denmark \\ ${ }^{5}$ Graduate School of Health, Discipline of Physiotherapy, University of Technology \\ Sydney, Sydney, New South Wales, Australia
}

\section{Twitter Peter William Stubbs @PeterStubbsPT}

Acknowledgements We would like to thank all contributing centres of their efforts with data collection. We would like to thank patients for contributing their time to answer the surveys and perform assessments.

Contributors Conception and design of the work: EN-S, PWS, DC, JFN and NBdVA. Acquisition of data: EN-S and N-BdVA. Analysis and interpretation of data: EN-S, PWS, DC, JFN and N-BdVA. Preparation of tables and figures: EN-S and PWS. Drafting the manuscript: EN-S and PWS. Editing revised manuscript: EN-S, PWS, DC, JFN and N-BdVA.

Funding This work was supported by 'Foundation of research, quality and education in practical physiotherapy' grant number A1334.

Competing interests None declared.

Patient consent for publication Not required.

Ethics approval The study was conducted in accordance with the Declaration of Helsinki and all included participants provided written and verbal informed consent. The study was assessed by the Regional Ethical Committee (ref. 2012-58-006) and approved by Regional Data Agency (j.nr. 1-16-02-757-17).

Provenance and peer review Not commissioned; externally peer reviewed.

Data availability statement The data that support the findings of this study are available from the corresponding author (EN), on reasonable request.

Supplemental material This content has been supplied by the author(s). It has not been vetted by BMJ Publishing Group Limited (BMJ) and may not have been peer-reviewed. Any opinions or recommendations discussed are solely those of the author(s) and are not endorsed by BMJ. BMJ disclaims all liability and responsibility arising from any reliance placed on the content. Where the content includes any translated material, BMJ does not warrant the accuracy and reliability of the translations (including but not limited to local regulations, clinical guidelines, terminology, drug names and drug dosages), and is not responsible for any error and/or omissions arising from translation and adaptation or otherwise.

Open access This is an open access article distributed in accordance with the Creative Commons Attribution Non Commercial (CC BY-NC 4.0) license, which permits others to distribute, remix, adapt, build upon this work non-commercially, and license their derivative works on different terms, provided the original work is properly cited, appropriate credit is given, any changes made indicated, and the use is non-commercial. See: http://creativecommons.org/licenses/by-nc/4.0/.

ORCID iD

Erhard Næss-Schmidt http://orcid.org/0000-0002-2539-0362

\section{REFERENCES}

1 Næss-Schmidt ET, Christiansen DH, Østgård RD, et al. Incidence of free of charge physiotherapy in a Danish national cohort of stroke, Parkinson's disease, multiple sclerosis and rheumatoid arthritis patients. Clin Epidemiol 2020;12:23-9.

2 McGrath R, Al Snih S, Markides K, et al. The burden of health conditions for middle-aged and older adults in the United States: disability-adjusted life years. BMC Geriatr 2019;19:1-13.

3 Pedersen BK, Saltin B. Exercise as medicine - evidence for prescribing exercise as therapy in 26 different chronic diseases. Scand J Med Sci Sports 2015;25 Suppl 3:1-72.

4 Amadid $\mathrm{H}$, Johansen NB, Bjerregaard $\mathrm{A}-\mathrm{L}$, et al. The role of physical activity in the development of first cardiovascular disease event: a tree-structured survival analysis of the Danish ADDITION-PRO cohort. Cardiovasc Diabetol 2018;17:1-12.

5 Pedersen BK. The physiology of optimizing health with a focus on exercise as medicine. Annu Rev Physiol 2019;81:607-27.

6 Morris J, Oliver T, Kroll T, et al. The importance of psychological and social factors in influencing the uptake and maintenance of physical activity after stroke: a structured review of the empirical literature. Stroke Res Treat 2012;2012:1-20.

7 Schmidt M, Schmidt SAJ, Sandegaard JL, et al. The Danish national patient registry: a review of content, data quality, and research potential. Clin Epidemiol 2015;7:449-90.

8 The Danish Health Authorities. Free of Charge Physioterapy [Internet], 2019. Available: https://www.sst.dk/da/udgivelser/2019/ vejledning-om-adgang-til-vederlagsfri-fysioterapi [Accessed $1 \mathrm{Sep}$ 2020].

9 World Health Organization. International Classification of Functioning, Disability and Health [Internet]. World Health Organization, Geneva, 2001. Available: https://apps.who.int/iris/bitstream/handle/10665/ 42407/9241545429.pdf [Accessed 1 Sep 2020].

10 Bennell K, Dobson F, Hinman R. Measures of physical performance assessments: Self-Paced Walk Test (SPWT), Stair Climb Test (SCT), Six-Minute Walk Test (6MWT), Chair Stand Test (CST), Timed Up \& Go (TUG), Sock Test, Lift and Carry Test (LCT), and Car Task. Arthritis Care Res 2011;63 Suppl 11:350-70.

11 Christopher A, Kraft E, Olenick H, et al. The reliability and validity of the timed up and go as a clinical tool in individuals with and without disabilities across a lifespan: a systematic review. Disabil Rehabil 2019:1-15.

12 Poulsen PH. Måleredskaber I klinisk praksis - Oversigt OG vurdering AF generiske måleredskaber SOM del AF en internetbaseret undersøgelsesmodel TIL vederlagsfrie patienter I fysioterapeutisk praksis 2013.

13 Janssen MF, Pickard AS, Golicki D, et al. Measurement properties of the EQ-5D-5L compared to the EQ-5D-3L across eight patient groups: a multi-country study. Qual Life Res 2013;22:1717-27.

14 Federici S, Bracalenti M, Meloni F, et al. World Health organization disability assessment schedule 2.0: an international systematic review. Disabil Rehabil 2017;39:2347-80.

15 Topp CW, Østergaard SD, Søndergaard S, et al. The WHO-5 well-being index: a systematic review of the literature. Psychother Psychosom 2015;84:167-76.

16 Johnsen SP, Ingeman A, Hundborg HH, et al. The Danish stroke Registry. Clin Epidemiol 2016;8:697-702.

17 Odgaard L, Poulsen I, Kammersgaard LP, et al. Surviving severe traumatic brain injury in Denmark: incidence and predictors of highly specialized rehabilitation. Clin Epidemiol 2015;7:225-34.

18 Stabel HH, Pedersen AR, Johnsen SP, et al. Functional independence: a comparison of the changes during 
neurorehabilitation between patients with nontraumatic subarachnoid hemorrhage and patients with intracerebral hemorrhage or acute ischemic stroke. Arch Phys Med Rehabil 2017;98:759-65.

19 Pedersen AR, Stubbs PW, Nielsen JF. Reducing redundant testing using the functional independence measure and early functional abilities scale during rehabilitation in patients with brain injury. Brain Inj 2018;32:1090-5.

20 Stubbs PW, Pallesen H, Pedersen AR, et al. Using EFA and FIM rating scales could provide a more complete assessment of patients with acquired brain injury. Disabil Rehabil 2014;36:2278-81.

21 Evensen KAl, Robinson HS, Meisingset I, et al. Characteristics, course and outcome of patients receiving physiotherapy in primary health care in Norway: design of a longitudinal observational project. BMC Health Serv Res 2018;18:1-10.

22 Meerhoff GA, van Dulmen SA, Maas MJM, et al. Development and evaluation of an implementation strategy for collecting data in a national registry and the use of patient-reported outcome measures in physical therapist practices: quality improvement study.. Phys Ther 2017;97:837-51.

23 Alexandria V. Physical Therapy Outcomes Registry [Internet]. American Physical Therapy Association, 2016. Available: http://www. ptoutcomes.com/Home.aspx [Accessed 1 Sep 2020].

24 Swinkels ICS, Hart DL, Deutscher D, et al. Comparing patient characteristics and treatment processes in patients receiving physical therapy in the United States, Israel and the Netherlands: cross sectional analyses of data from three clinical databases. BMC Health Serv Res 2008;8:1-12.

25 Murtagh KN, Hubert HB. Gender differences in physical disability among an elderly cohort. Am J Public Health 2004;94:1406-11.
26 Whitacre CC, Reingold SC, O'Looney PA, et al. A gender gap in autoimmunity. Science 1999;283:1277-8.

27 Næss-Schmidt E, Pedersen A, Christiansen D, et al. Daily activity and functional performance in people with chronic disease : A crosssectional study. Cogent Med 2020;7:1-13.

28 Munce SEP, Stewart DE. Gender differences in depression and chronic pain conditions in a national epidemiologic survey. Psychosomatics 2007;48:394-9.

29 Celius EG, Smestad C. Change in sex ratio, disease course and age at diagnosis in Oslo MS patients through seven decades. Acta Neurol Scand Suppl 2009;120:27-9.

30 Nutt JG, Wooten GF. Diagnosis and initial management of PD. N Engl J Med 2005;7:951-64.

31 Andersen KK, Andersen ZJ, Olsen TS. Age- and gender-specific prevalence of cardiovascular risk factors in 40,102 patients with first-ever ischemic stroke: a nationwide Danish study. Stroke 2010;41:2768-74.

32 Dorsey ER, Bloem BR. The Parkinson Pandemic-A call to action. JAMA Neurol 2018;75:9-10.

33 Farag I, Howard K, Hayes AJ, et al. Cost-Effectiveness of a homeexercise program among older people after hospitalization. J Am Med Dir Assoc 2015;16:490-6.

34 Russell TG. Telerehabilitation: a coming of age. Aust J Physiother 2009;55:5-6.

35 Budtz CR, Mose S, Christiansen DH. Socio-Demographic, clinica and psychological predictors of healthcare utilization among patients with musculoskeletal disorders: a prospective cohort study. BMC Health Serv Res 2020;20:1-12. 